\title{
Harmonic Elimination for Multilevel Converter with Programmed PWM Method
}

\author{
Zhong Du, Leon M. Tolbert, John N. Chiasson \\ The University of Tennessee \\ Department of Electrical and Computer Engineering \\ Knoxville, Tennessee 37996-2100 \\ E-mail: zdu1@utk.edu, tolbert@utk.edu, chiasson@utk.edu
}

\begin{abstract}
This paper presents a programmed PWM method to eliminate specific higher order harmonics of multilevel converters. First, resultant theory is applied to transcendental equations to eliminate low order harmonics and to determine switching angles for the fundamental frequency switching techniques. Next the magnitudes and phases of the residual higher order harmonics are computed, generated, and subtracted from the original voltage waveform to eliminate these higher order harmonics. The simulation results show that the method can effectively eliminate the specific harmonics, and a low total harmonic distortion (THD) near sine wave is produced. An experimental 11-level $\mathrm{H}$-bridge multilevel converter with a firston, first-off switching strategy, which is used to balance loads between several levels, is employed to validate the method. The experimental results show that the method can effectively eliminate the specific harmonics, and the output voltage waveforms have less THD than that from the fundamental frequency switching techniques.
\end{abstract}

Keywords- Multilevel converter, programmed PWM, harmonic elimination.

\section{INTRODUCTION}

Multilevel converters have received more and more attention because of their capability of high voltage operation, high efficiency, and low electromagnetic interference (EMI) [3][6]. The desired output of a multilevel converter is synthesized by several sources of DC voltages. With an increasing number of DC voltage sources, the converter voltage output waveform approaches a nearly sinusoidal waveform while using a fundamental frequency switching scheme. This results in low switching losses, and because of several DC sources, the switches experience a lower $d V / d t$. As a result, the multilevel converter technology is a promising technology for high power electric devices such as utility applications. For these applications, the output voltage of the converters must meet maximum THD limitations such as those specified in IEEE 519 [5]. Therefore, some kind of method must be used to control the harmonics.

The transcendental equations characterizing the harmonic content can be converted into polynomial equations. Elimination theory $[1][2][4]$ (using resultants) has been employed to determine the switching angles to eliminate specific harmonics, such as 5th, 7th, 11th, and the 13th. However, as the number of DC sources increases, the degrees of the polynomials in these equations are large and one reaches the limitations of contemporary computer algebra software tools (e.g., Mathematic or Maple) to solve the system of polynomial equations using elimination theory [8].

Due to the computation difficulty of the resultant method with high degrees, a new programmed PWM algorithm is proposed in this paper to eliminate higher order harmonics. First, the low order harmonics (e.g., the 5th, 7th, 11th and 13th) can be eliminated using a fundamental frequency switching scheme in which the switch angles are determined using elimination theory. Next, specifically chosen higher harmonics (e.g., the odd non triplen harmonics from the 19th to the 49th in the experiments) are eliminated by using an additional switching angle (one for each higher harmonic) to generate the negative of the harmonic to cancel it.

An experimental 11-level H-bridge multilevel converter with a first-on, first-off switching strategy (used to balance loads between several DC sources), is employed to validate the method. The experimental results show that the method can effectively eliminate the specific harmonics, and the output voltage waveform has much less THD than that from the fundamental switching techniques.

The programmed PWM method also offers flexibility between switching frequency and higher order harmonic elimination. In order to eliminate more harmonics than the number of levels in a multilevel converter, the switching frequency must be higher, however.

\section{RESUltANT METHOD FOR MULTILEVEL CONVERTER}

A multilevel converter uses several DC sources to synthesize a sinusoidal wave. Thus, the control of the multilevel converter is to choose a series of switching angles to synthesize a desired sinusoidal voltage waveform. The 11level multilevel converter output voltage waveform generated by fundamental frequency switching scheme is shown in Fig. 1 .

The Fourier series expansion of the output voltage waveform using fundamental frequency switching scheme as shown in Fig. 1 is as follows:

$$
\begin{aligned}
& V(\omega t)= \\
& \sum_{n=1,3,5, \ldots}^{\infty} \frac{4 V_{d c}}{n \pi}\left(\cos \left(n \theta_{1}\right)+\cos \left(n \theta_{2}\right)+\cos \left(n \theta_{3}\right)+\ldots+\cos \left(n \theta_{s}\right)\right) \sin (n \omega t)
\end{aligned}
$$




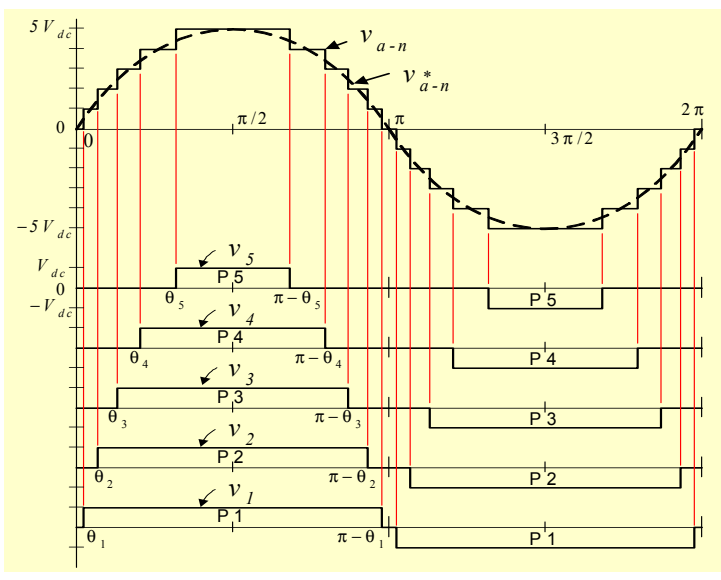

Fig. 1. Output waveform of multilevel converter using fundamental frequency switching scheme

where $s$ is the number of DC sources in a cascaded H-bridge multilevel converter. Ideally, given a desired fundamental voltage $V_{l}$, one wants to determine the switching angles $\theta_{l}, \cdots$, $\theta_{S}$ so that $V(\omega t)=V_{l} \sin (\omega t)$, and specific higher harmonics of $V(n \omega t)$ are equal to zero. For a three-phase application, the triplen harmonics in each phase need not be cancelled as they automatically cancel in the line-to-line voltages. For example, in the case of $s=5 \mathrm{DC}$ sources, the 5th, 7th, 11th, and 13th order harmonics can be cancelled.

The switching angles can be found by solving the following equations:

$$
\begin{aligned}
& \cos \left(\theta_{1}\right)+\cos \left(\theta_{2}\right)+\cos \left(\theta_{3}\right)+\cos \left(\theta_{4}\right)+\cos \left(\theta_{5}\right)=m \\
& \cos \left(5 \theta_{1}\right)+\cos \left(5 \theta_{2}\right)+\cos \left(5 \theta_{3}\right)+\cos \left(5 \theta_{4}\right)+\cos \left(5 \theta_{5}\right)=0 \\
& \cos \left(7 \theta_{1}\right)+\cos \left(7 \theta_{2}\right)+\cos \left(7 \theta_{3}\right)+\cos \left(7 \theta_{4}\right)+\cos \left(7 \theta_{5}\right)=0 \\
& \cos \left(11 \theta_{1}\right)+\cos \left(11 \theta_{2}\right)+\cos \left(11 \theta_{3}\right)+\cos \left(11 \theta_{4}\right)+\cos \left(11 \theta_{5}\right)=0 \\
& \cos \left(13 \theta_{1}\right)+\cos \left(13 \theta_{2}\right)+\cos \left(13 \theta_{3}\right)+\cos \left(13 \theta_{4}\right)+\cos \left(13 \theta_{5}\right)=0
\end{aligned}
$$

where the modulation index $m$ is defined as $m=\pi V_{l} /\left(4 V_{d c}\right)$.

The transcendental equations characterizing the harmonic content can be converted into polynomial equations. Then the resultant method is employed to find the solutions when they exist. The advantage of the resultant method is that it can find all the solutions for the equations [7][8]. The 11-level solutions with lowest THD are shown in Fig. 2. The THD for the corresponding solution is shown in Fig. 3.

From the solution shown in Fig. 2, it can be derived that the solutions for $m$ exist in a range from 1.88 to 4.23 . It also can be derived that the solutions are not continuous. For some modulation indices, there are no corresponding solutions. There are more than one set for some modulation indices; Fig. 2 shows only the lowest THD solution set.

\section{PROPOSED HARMONIC ELIMINATION METHOD}

From equation (1), the voltage content can be divided into four parts:

$$
V(\omega t)=V_{p 1}(t)+V_{p 2}(t)+V_{p 3}(t)+V_{p 4}(t)
$$

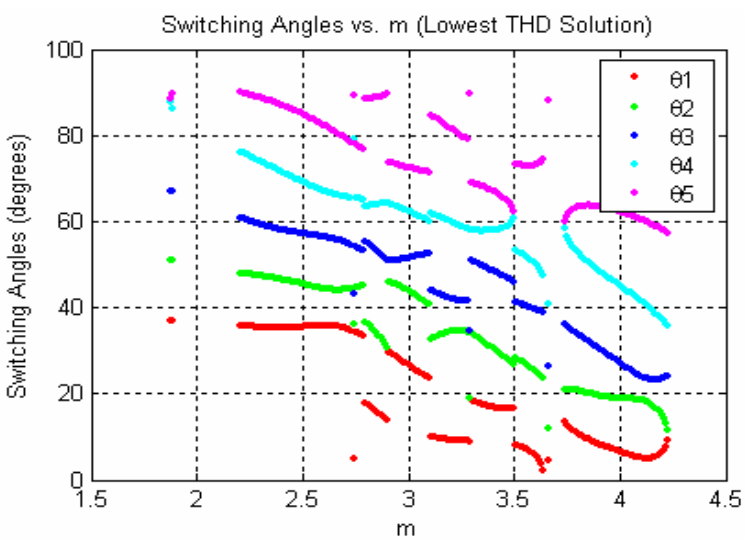

Fig. 2. Solutions for switching angles with lowest THD vs. $m$

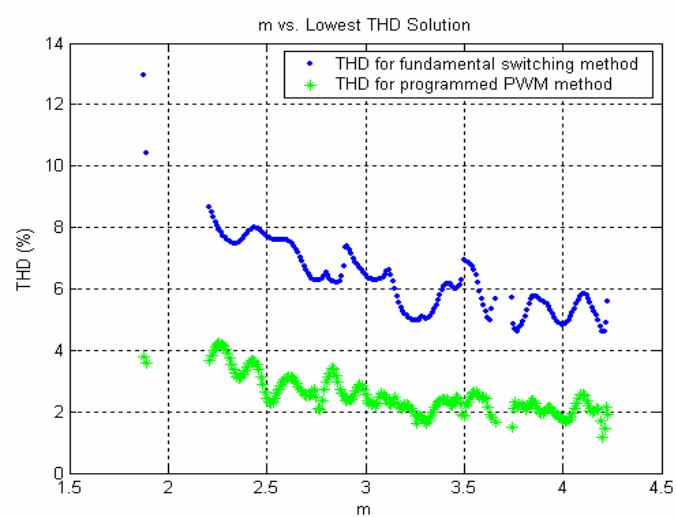

Fig. 3. THDs for fundamental frequency switching method and programmed PWM method vs. $m$

1. Fundamental frequency voltage:

$V_{p 1}(t)=\frac{4 V_{d c}}{\pi}\left[\cos \left(\theta_{1}\right)+\cos \left(\theta_{2}\right)+\cdots+\cos \left(\theta_{s}\right)\right] \sin (\omega t)$

2. Triplen harmonic voltages:

$V_{p 2}(t)=\frac{4 V_{d c}}{n \pi} \prod_{n=3,9,15, \cdots}\left[\cos \left(n \theta_{1}\right)+\cos \left(n \theta_{2}\right)+\cdots+\cos \left(n \theta_{s}\right)\right] \sin (n \omega t)$

3. Low order harmonic voltages that can be eliminated by applying the resultant method.

$$
V_{p 3}(t)=\frac{4 V_{d c}}{n \pi} \prod_{n=5,7,11,13}\left[\cos \left(n \theta_{1}\right)+\cos \left(n \theta_{2}\right)+\cdots+\cos \left(n \theta_{s}\right)\right] \sin (n \omega t)
$$

4. High order harmonic voltages that cannot be eliminated by applying the resultant method.

$$
V_{p 4}(t)=\frac{4 V_{d c}}{n \pi} \prod_{n=17,19,23, \cdots}\left[\cos \left(n \theta_{1}\right)+\cos \left(n \theta_{2}\right)+\cdots+\cos \left(n \theta_{s}\right)\right] \sin (n \omega t)
$$


Using the resultant method of [7][8], (6) is eliminated using a fundamental frequency switching scheme. Assuming the application is a balanced three-phase system, (5) need not be eliminated. This then leaves (7). To eliminate these harmonics, a square wave is generated (one for each of these harmonics) whose fundamental is equal to the negative of the harmonic that is to be eliminated. For example, to eliminate the 17th harmonic, a square wave whose Fourier series expansion is

$$
V_{k_{1}}(t)=-\frac{4 V_{d c}}{m \pi} \prod_{m=1,3,5,7, \cdots}\left[\cos \left(m k_{1} \theta_{1}\right)+\cos \left(m k_{1} \theta_{2}\right)+\cdots+\cos \left(m k_{1} \theta_{s}\right)\right] \sin \left(m k_{1} \omega t\right)
$$

is generated. The $m=1$ and $k_{l}=17$ term of (8) cancels the $n=17$ term of (7) and the next harmonic of concern that is produced by (8) is at $5 \times 17=85$. This harmonic and higher ones $(7 \times 17$, etc.) are easy to filter using a low-pass filter. Repeating the above procedure to the multilevel converter, the 19th, 23rd ... 49th harmonics can all be eliminated. The net effect of this method is to remove the low order harmonics and to generate new higher order harmonics by increasing the switching frequency. The $T H D=\frac{\sqrt{V_{5}^{2}+V_{7}^{2}+\cdots+V_{79}^{2}}}{V_{1}}$ of the output voltage versus $m=\pi V_{1} /\left(4 V_{d c}\right)$ is shown in Fig. 3 .

In theory, to eliminate the $n$th harmonic, the multilevel converter needs to switch $n$ times in a cycle. So the switching number in a cycle for the programmed PWM harmonic elimination method is $N_{s w} \leq \underset{n=17,19,23, \cdots}{i}$, where $n$ is the harmonic number.

If a harmonic is near zero, the control resolution is lower than that required to eliminate the harmonic, and switching will not occur. For this paper, no attempt was made to eliminate residual harmonics with amplitudes less than $0.5 \%$.

If the switching control resolution is set, the number of switching can be computed by:

$$
N_{s w}=\prod_{h_{i} \in\{17,19,23, \cdots\}} h_{i}
$$

where $h_{i}$ is the harmonic number of those harmonics chosen for elimination (i.e. harmonics whose amplitudes are greater than $0.5 \%$ of the fundamental).

Another case is the overlap, if one switching off time follows a switching on time immediately, then the switch will be on until next switching off occurs. This will decrease the number of switching during each period. It is not easy to analytically compute this second case. However, in simulation, the number of switching can be counted.

If the THD limit is set to $5 \%$ and the control resolution is set to $0.5 \%$, the switching number computed for one cycle by (9) is shown in Fig. 4. From Fig. 4, it can be derived that to eliminate harmonics such that the THD of the output voltage is below $5 \%$, the switching number is below 50 for half of the modulation range; it is below 150 for most of the modulation range.

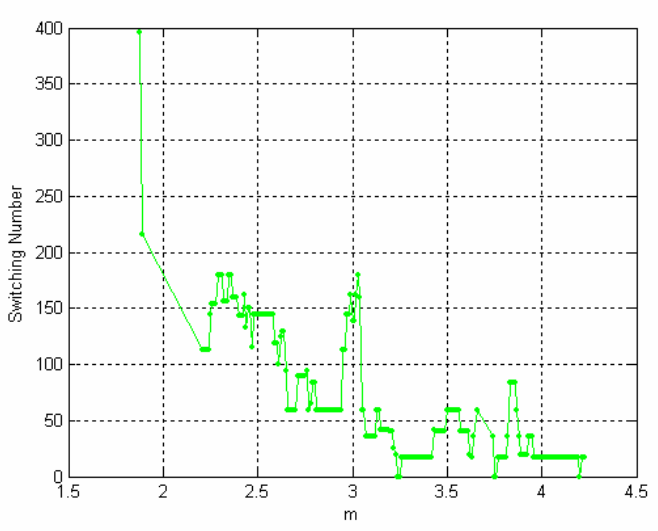

Fig. 4. Switching number for a cycle for 5\% THD

Instead of limiting the THD of the output voltage, the goal can be to eliminate certain harmonics in the output voltage. For example, in the system, if the harmonics will be eliminated up to 31 st, then the switching number for one cycle and its corresponding THD are shown in Fig. 5. It can been seen that if we eliminate the harmonics up to 31 st, the THD will be below $5 \%$ except for a few low modulation indices.

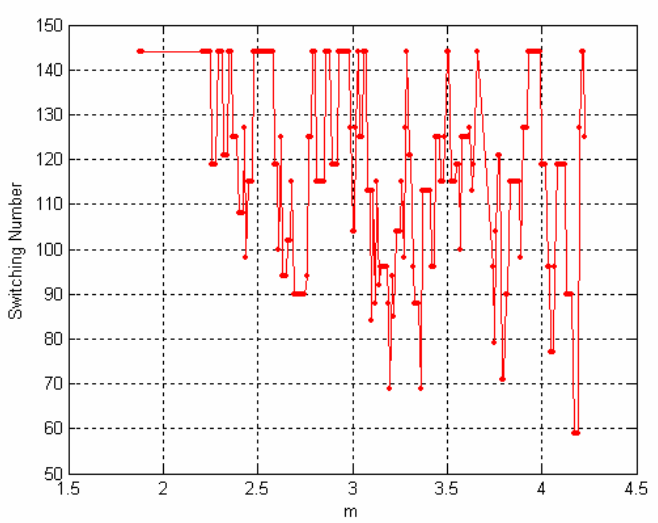

(a)

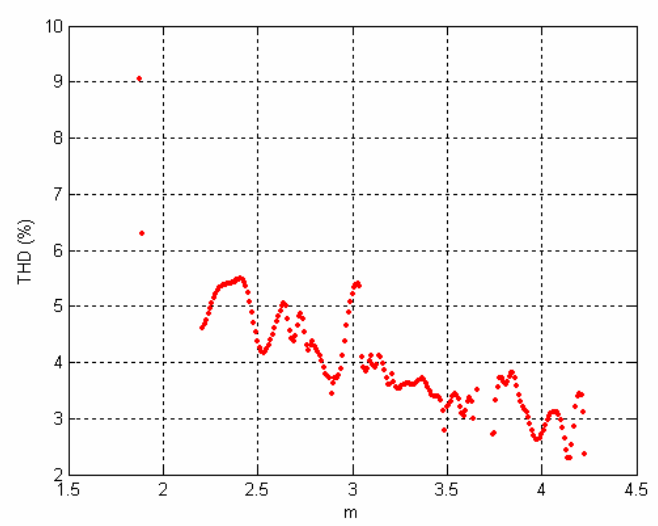

(b)

Fig. 5. (a) Switching number for a cycle to eliminate harmonics up to 31 st; (b) its corresponding THD 
Here, the switching number in a cycle determines the effective switching frequency. For example, if the number of switchings is 50 for a multilevel converter, and the desired output frequency is $60 \mathrm{~Hz}$ which will be synthesized by $5 \mathrm{DC}$ sources, then the effective switching frequency is $50 \times 60=$ $3,000 \mathrm{~Hz}$; however, the switching frequency for each switch is only $50 \times 60 / 5=600 \mathrm{~Hz}$.

As it is seen from (1), the harmonic spectrum of the proposed method is linearly attenuated by the order of the harmonics. For a traditional PWM method, the harmonics in the converter output voltage waveform appear as sidebands, centered around the switching frequency and its multiples [9]. The traditional PWM method can not completely eliminate the specified low order harmonics. The proposed programmed PWM method here can completely eliminate all the specified harmonics. This programmed PWM method can also be called computed PWM method since its pulses are computed.

To verify the proposed harmonic elimination method, a simulation case with $m=2.28$ is implemented. The line-line voltage simulation for this case of $m=2.28$ and its normalized FFT analysis are shown in Fig. 6. As expected, the line-line voltage has no triplen harmonics and the first non-zero harmonic is the 53rd harmonic.

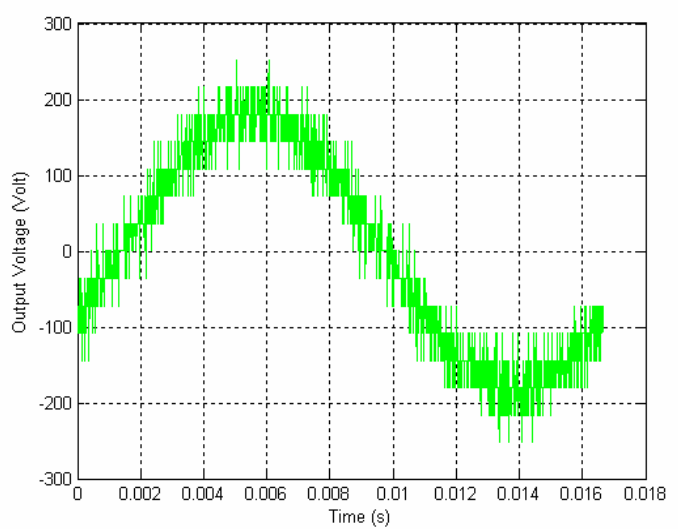

(a)

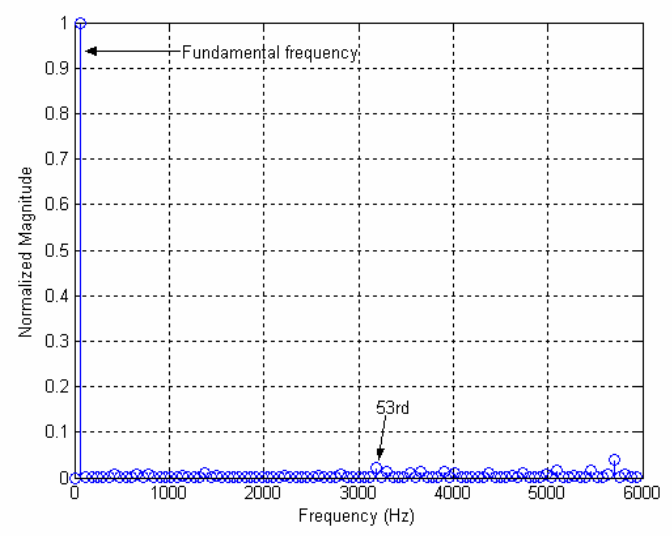

(b)

Fig. 6. (a) Simulation line-line voltage; (b) its corresponding normalized FFT analysis for harmonic elimination up to 49th and $m=2.28$

\section{SWITCHING CONTROL STRATEGY}

To balance the switching loss during a whole cycle, the firston, first-off strategy is employed to control the switching here.

The idea for first-on, first-off strategy is to turn on the switches and turn off the switches in turn. Therefore this strategy distributes the switching times between several levels, so each level has about the same average switching time in a cycle. This strategy can also balance the load between several levels.

The control flow chart is shown in Fig. 7. The strategy works as follows. If the next output voltage is higher than the previous voltage, the strategy picks the next available switch in the switch table and turns it on. Repeat this process until the output voltage reaches the desired voltage. A similar process will occur when the next output voltage is lower than the previous voltage. Repeat this process until the voltage for a whole cycle has been synthesized.

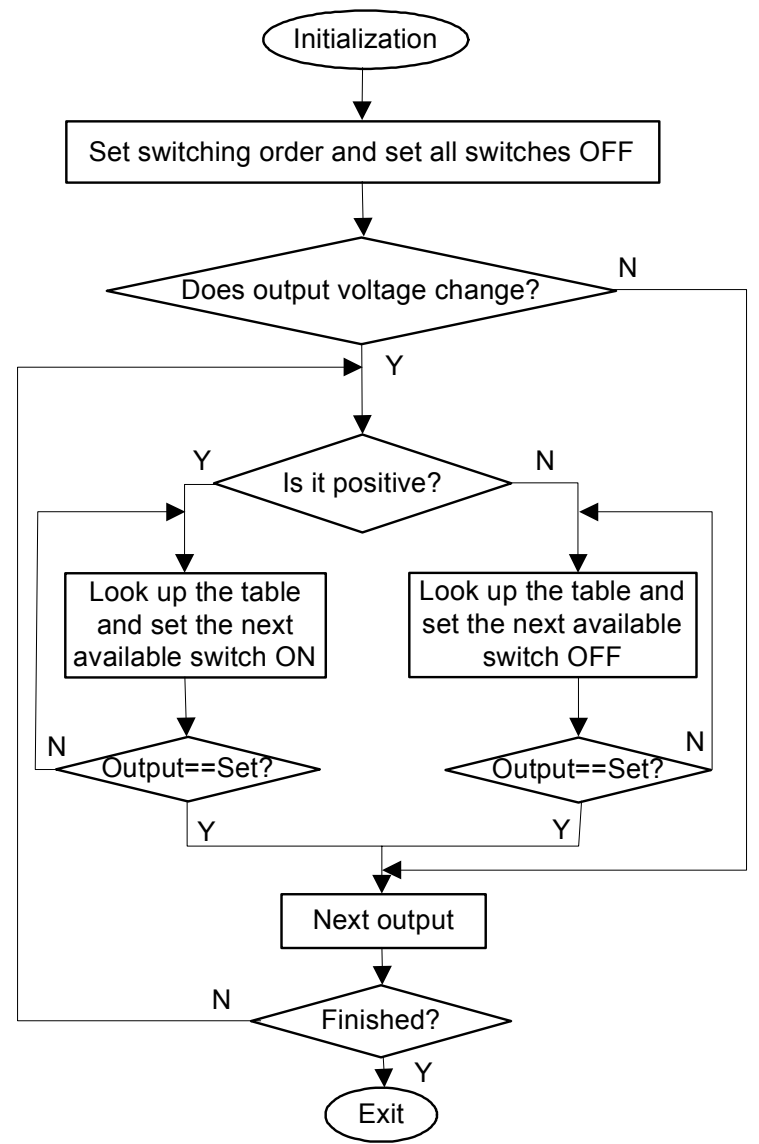

Fig. 7. Control flow chart for first on, first off strategy

\section{EXPERIMENTAL RESULTS}

The proposed programmed PWM method has been implemented in an 11-level (5 DC sources) H-bridge multilevel converter shown in Fig. 8 to eliminate harmonics below the 50th. 


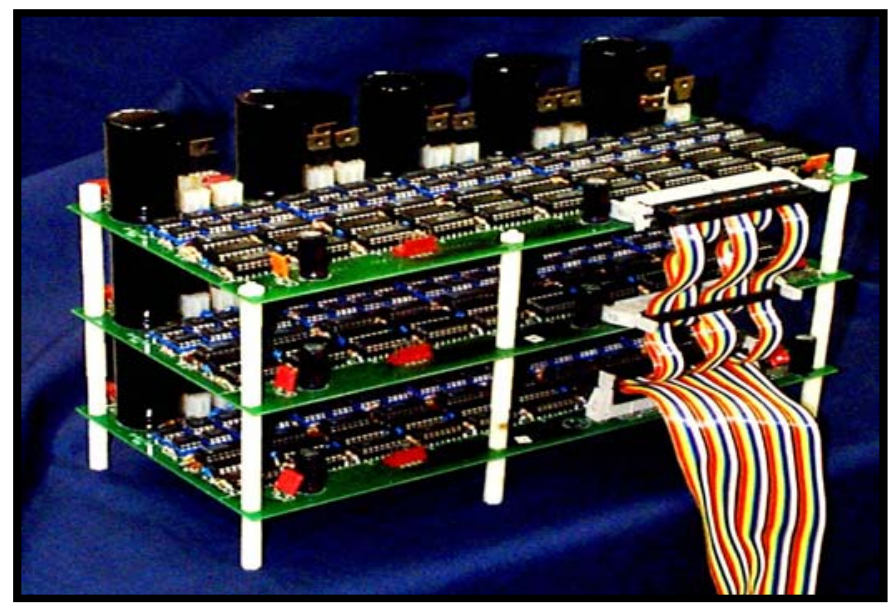

Fig. $8.10 \mathrm{~kW}$ multilevel converter prototype

This prototype converter has been built using $60 \mathrm{~V}, 100 \mathrm{~A}$ MOSFETs as the switching devices. In the experimental study, this prototype system was configured to have a nominal DC link of 36 Volts for each cell. This converter was then used to validate the theoretical computation results and simulation results for the programmed harmonic elimination method.

A real-time controller based on an Altera FLEX 10K field programmable gate array (FPGA), which has a $8 \mu$ s resolution was used to implement the algorithm.

Fig. 9 shows an example with $m=2.28$ of $60-\mathrm{Hz}$ phase output voltage waveforms and line-line voltage waveform. Fig. 9(c) shows the FFT analysis of the line-line voltage.

From the FFT plot in Fig. 9(c), it is seen that all harmonics below the 50th have been eliminated. The first non-zero harmonic voltage is the $53 \mathrm{rd}$. This is consistent with both the theoretical computation and simulation.

A $10 \mu \mathrm{F}$ capacitor was added to the output of the converter as a filter, and the output line-line voltage looked as shown in Fig. 10(a). Its normalized FFT analysis is shown in Fig. 10(b). It can be seen from Fig. 10 that the output voltage is very near a sinusoidal waveform.

From the FFT analysis in Fig. 10(b), it is obvious that all the higher order harmonics are zero. The THD is from low order harmonics. This validated the expectation that a small filter can eliminate residual higher order harmonics, and a low THD voltage can be achieved easily.

Other experiments were also implemented. The THD computation comparing the fundamental frequency switching scheme and the programmed harmonic elimination scheme proposed here under different situations are given in Table I.

From the results shown in Table I, it can be derived that the proposed programmed harmonic elimination method can decrease the THD of an output voltage generated by the fundamental frequency switching scheme. The experiments correspond very well with the theoretical computation and simulation.

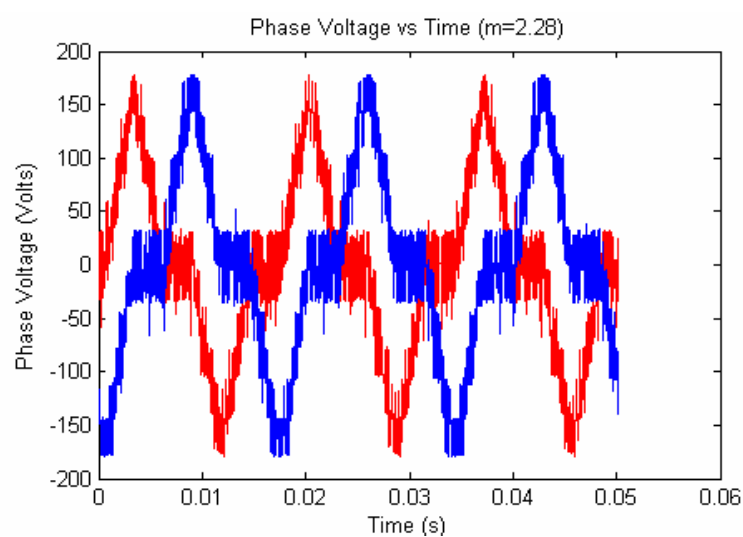

(a)

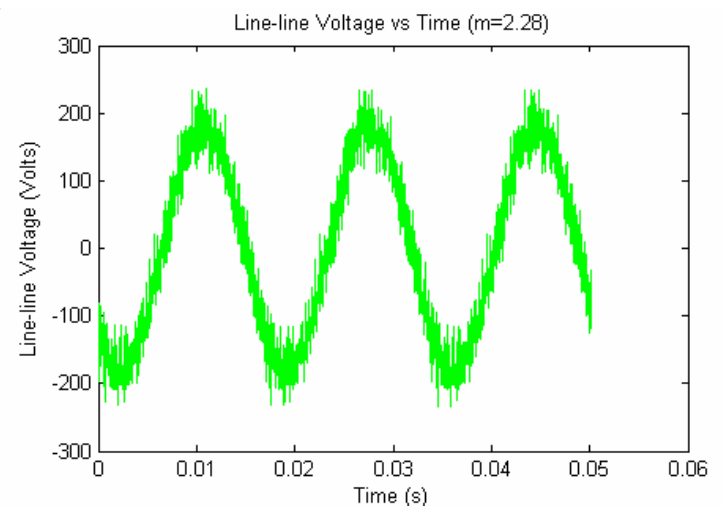

(b)

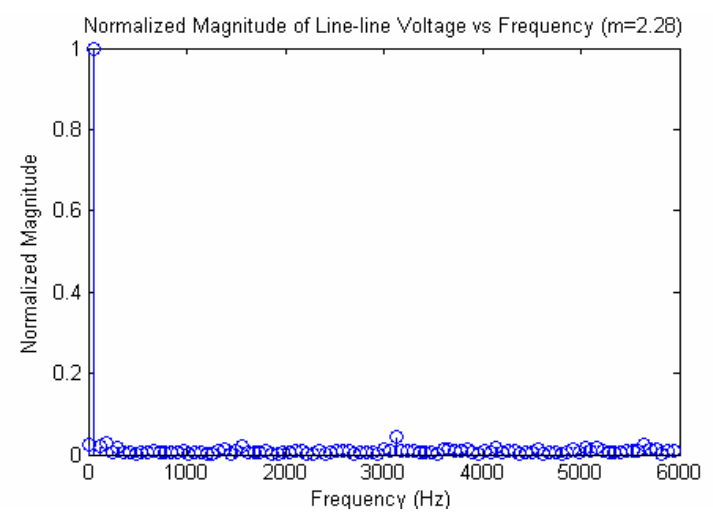

(c)

Fig. 9. (a) Phase voltage of experiment with programmed PWM method; (b) line-line voltage of experiment with programmed PWM method; (c) normalized FFT analysis of line-line voltage. $(m=2.28)$

From the previous discussion, it can be seen that if lower THD is desired, more switchings are needed. However more switchings will result in higher switching loss and low efficiency. Therefore for a specific application, there is a tradeoff between THD and efficiency. As this is a multilevel converter, the effective switching frequency can be higher than the actual switching frequency of individual devices. 


\section{CONCLUSION}

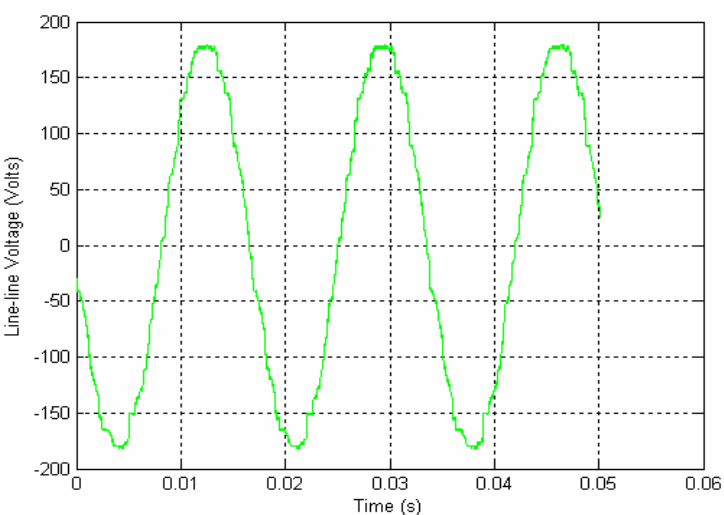

(a)

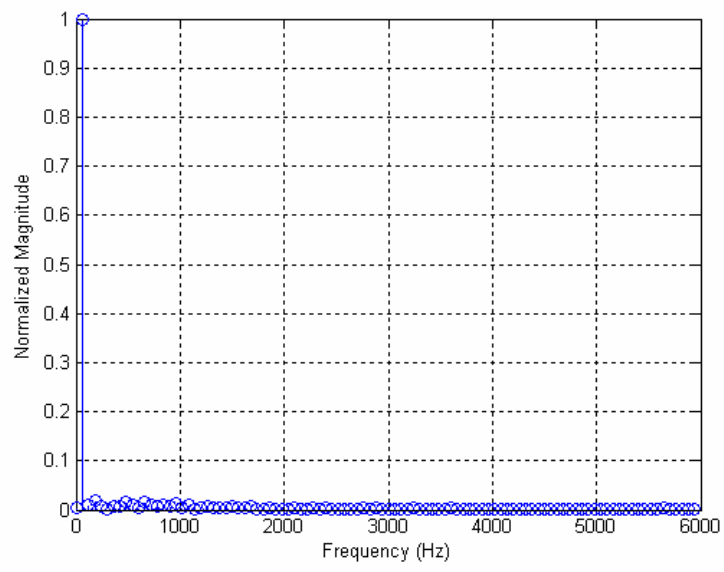

(b)

Fig. 10. (a) Line-line voltage with a $10 \mu \mathrm{F}$ capacitor filter $(m=2.28$ )

(b) normalized FFT analysis of line-line voltage shown in (a)

Table I. THD* $(m=2.28)$ under different conditions

\begin{tabular}{|c|c|}
\hline Method & THD (\%) \\
\hline $\begin{array}{c}\text { Fundamental switching } \\
\text { frequency method }\end{array}$ & 7.93 \\
\hline $\begin{array}{c}\text { Theoretical computation for } \\
\text { programmed PWM method }\end{array}$ & 4.15 \\
\hline Simulation & 4.31 \\
\hline $\begin{array}{c}\text { Experimental result 1 } \\
(f=30 \mathrm{~Hz} \text { and no filter })\end{array}$ & 4.57 \\
\hline $\begin{array}{c}\text { Experimental result 2 } \\
(f=60 \mathrm{~Hz} \text { and no filter })\end{array}$ & 4.08 \\
\hline $\begin{array}{c}\text { Experimental result 3 } \\
\left(\begin{array}{c}\mathrm{f}=\mathrm{Hz} \text { and with a } 10 \\
\mu F \text { capacitor filter })\end{array}\right.\end{array}$ & 2.26 \\
\hline
\end{tabular}

*THD computation is up to the 80th harmonic.
A programmed PWM algorithm has been proposed and developed to eliminate specific harmonics for multilevel converters. The simulation results and experimental results show that the algorithm can be used to eliminate specific higher order harmonics effectively and result in a dramatic decrease in the THD.

\section{ACKNOWLEDGMENTS}

We would like to thank the National Science Foundation for partially supporting this work through contract NSF ECS0093884. We would also like to thank Oak Ridge National Laboratory for partially supporting this work through UT/Battelle contract No. 400023754.

\section{REFERENCES}

[1] H. S. Patel and R. G. Hoft, "Generalized harmonic elimination and voltage control in thyristor inverters: Part I -harmonic elimination," IEEE Transactions on Industry Applications, vol. 9, May/June 1973, pp. 310-317.

[2] H. S. Patel and R. G. Hoft, "Generalized harmonic elimination and voltage control in thyristor inverters: Part II -voltage control technique," IEEE Transactions on Industry Applications, vol. 10, Sept./Oct. 1974, pp. 666-673.

[3] J. S. Lai and F. Z. Peng, "Multilevel converters - A new breed of power converters," IEEE Transactions on Industry Applications, vol. 32, no. 3, May /June 1996, pp. 509-517.

[4] P. N. Enjeti, P. D. Ziogas, J. F. Lindsay, "Programmed PWM techniques to eliminate harmonics: A critical evaluation" IEEE Transactions on Industry Applications, vol. 26, no. 2, March/April. 1990. pp. $302-316$.

[5] C. K. Duffey, R. P. Stratford, "Update of harmonic standard IEEE-519: IEEE recommended practices and requirements for harmonic control in electric power systems," IEEE Transactions on Industry Applications, vol. 25, no. 6, Nov./Dec. 1989, pp. 1025-1034.

[6] L. M. Tolbert, F. Z. Peng, T. G. Habetler, "Multilevel converters for large electric drives, "IEEE Transactions on Industry Applications, vol. 35, no. 1, Jan./Feb. 1999, pp. 36-44.

[7] J. N. Chiasson, L. M. Tolbert, K. J. McKenzie, Z. Du, "Control of a multilevel converter using resultant theory, "IEEE Transactions on Control System Theory, vol. 11, no. 3, May 2003, pp. 345-354.

[8] J. N. Chiasson, L. M. Tolbert, K. J. McKenzie, Z. Du, "A new approach to solving the harmonic elimination equations for a multilevel converter," IEEE Industry Applications Society Annual Meeting, October 12-16, 2003, Salt Lake City, Utah, pp. 640-645.

[9] N. Mohan, T. M. Undeland, and W. P. Robbins, Power Electronics: Converters, Applications, and Design, Third Edition. John Wiley and Sons, 2003. 